\title{
Carcinoma de células pequeñas de cérvix, presentación de caso
}

\section{Small Cell Carcinoma of the uterine cervix, about a case}

1 Mildrey García Hernández

iD http://orcid.org/0000-0002-7247-1384

Hospital Universitario Comandante Faustino Pérez Hernández, Matanzas, Cuba mildreyg.mtz@infomed.sld.cu

2 Ihosvannys Enrique Carreño Rolando (iD) http://orcid.org/0000-0003-1259-3491 Hospital Universitario Comandante Faustino Pérez Hernández, Matanzas, Cuba ihosvanny.mtz@infomed.sld.cu

3 Luís Enrique Curbelo Gutiérrez (iD) http://orcid.org/0000-0002-7761-9595 Hospital Universitario Comandante Faustino Pérez Hernández, Matanzas, Cuba luis.curbelo@icloud.com

\section{Carcinoma de células pequeñas de cérvix, presentación de caso. Anatomía Digital, 5(1), \\ Cítese: $\quad 37-48$. https://doi.org/10.33262/anatomiadigital.v5i1.1968}

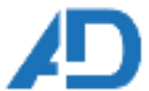

Ciencia Digital

ANATOMÍA DIGITAL, es una Revista Electónica, Trimestral, que se publicará en soporte electrónico tiene como misión contribuir a la formación de profesionales competentes con visión humanística y crítica que sean capaces de exponer sus resultados investigativos y científicos en la misma medida que se promueva mediante su intervención cambios positivos en la sociedad. https://anatomiadigital.org

La revista es editada por la Editorial Ciencia Digital (Editorial de prestigio registrada en la Cámara Ecuatoriana de Libro con No de Afiliación 663) www.celibro.org.ec

Esta revista está protegida bajo una licencia Creative Commons AttributionNonCommercialNoDerivatives 4.0 International. Copia de la licencia: http://creativecommons.org/licenses/by-nc-nd/4.0/ 


\section{Palabras}

claves: Cáncer de cuello uterino, tumor neuroendocrino, carcinoma de células pequeñas cervicales.

\section{Keywords:}

Cervical

cancer, neuroendocrine tumor, cervical small cell carcinoma

\section{Resumen}

Introducción: El cáncer de cuello uterino es una de las principales causas de muerte por cáncer entre las mujeres. Los tumores neuroendocrinos son agresivos y se desarrollan a partir de células endocrinas en varios órganos, incluido el tracto genital femenino, y específicamente el cuello uterino. Objetivo: describir las características clínico- epidemiológicas del carcinoma de células pequeñas. Metodología: hicimos una revisión de la bibliografía sobre el tema y presentamos los resultados. Resultados: Representan entre el $0,9 \%$ y el $1,5 \%$ de todos los tumores del cuello uterino y generalmente afectan a las mujeres en edad reproductiva. Dada la rareza de la enfermedad, actualmente se dispone de datos limitados sobre la biología, el comportamiento clínico y el manejo de estos tumores agresivos. La asociación etiopatogénica entre el carcinoma de células pequeñas de cérvix (SCCC) y las infecciones por VPH de alto riesgo ha sido documentada en algunos estudios y tiene un pronóstico mucho peor en comparación con otros tipos histológicos de carcinoma cervical. Las modalidades de tratamiento del SCCC y del cáncer de pulmón de células pequeñas son similares debido a un comportamiento biológico similar. La quimioterapia es un componente importante de la terapia multimodal. Hicimos una presentación de caso de un paciente con diagnóstico de SCCC con una supervivencia global de seis años, muy por encima de la supervivencia internacional promedio. Conclusiones: deben continuar las investigaciones para llegar a un consenso sobre el tratamiento adecuado.

\section{Abstract}

Introduction: Cervical cancer is one of the leading causes of cancer death among women. Neuroendocrine tumors are aggressive and develop from endocrine cells in various organs, including the female genital tract, and specifically the uterine cervix. Objective: to describe the clinical- epidemiological characteristics of small cell carcinoma. Methodology: we made a review of the bibliography on the subject and presented the results. Results: They account for $0.9 \%-1.5 \%$ of all tumors of the uterine cervix and generally affect women of reproductive age. Given the rarity of the disease, limited data are currently available regarding 
the biology, clinical behavior, and management of such aggressive tumors. The etiopathogenetic association between cervical small cell carcinoma (SCCC) and high- risk HPV infections has been well documented in some studies and has a much worse prognosis compared with other histological types of cervical carcinoma. The treatment modalities of SCCC and small-cell lung cancer are similar due to similar biological behavior. Chemotherapy is an important component of multimodal therapy. We made a case presentation of a patient with diagnosis of SCCC with an overall survival of six years, well above the average international survival. Conclusions: research should continue to reach consensus on the appropriate treatment.

\section{Introducción.}

El cáncer de cuello uterino es una de las principales causas de muerte por cáncer entre las mujeres. En todo el mundo, el cáncer de cuello uterino es la cuarta neoplasia maligna que ocurre con más frecuencia en las mujeres, y se estima que se presenten 530.000 nuevos casos anuales con 270.000 muertes. Aproximadamente el $85 \%$ de las muertes mundiales por cáncer de cuello uterino se producen en países subdesarrollados o en vías de desarrollo, y la tasa de mortalidad es 18 veces mayor en los países de ingresos bajos y medianos en comparación con los países más ricos. Las tasas de incidencia más altas se producen en América Central y del Sur, el Caribe, África subsahariana y el sur de Asia. En los Estados Unidos en 2016, se estima que hubo 12.990 casos y 4120 muertes por cáncer de cuello uterino, y la mediana de edad en el momento del diagnóstico fue de 47 años. ${ }^{(1)}$

La identificación de factores de riesgo desempeña un papel fundamental en la prevención del cáncer de cuello uterino. Numerosos estudios han demostrado la asociación entre varios factores de riesgo y el cáncer. ${ }^{(2,3)}$ Se ha notificado una asociación entre la tasa de supervivencia al cáncer de cuello uterino y la situación socioeconómica de las mujeres. ${ }^{(4)}$ Además, también se han identificado como factores de riesgo enfermedades venéreas, factores reproductivos, anticonceptivos orales a largo plazo y cuestiones de comportamiento como el tabaquismo y la obesidad. ${ }^{(5)}$ Las infecciones por el virus del papiloma humano (VPH) de alto riesgo se han establecido como el principal factor de riesgo para el desarrollo del cáncer de cuello uterino y se ha 
declarado que el VPH 16 y 18 son la causa del $71 \%$ de los casos de cáncer de cuello uterino en el continente africano. ${ }^{(6)}$

El tratamiento del cáncer de cuello uterino se determina tradicionalmente por el estadio y la extensión de la enfermedad. Hay esencialmente tres tipos de tratamientos estándar contra el cáncer de cuello uterino, incluyendo cirugía, radiación y quimioterapia. El tratamiento quirúrgico consiste una histerectomía radical con linfadenectomía pélvica. $(7,8)$

La radioterapia intenta destruir las células cancerosas y se administra en las fases avanzadas del cáncer de cuello uterino con intensión curativa o radical, puede administrarse de forma externa (teleterapia) o interna (braquiterapia) y pueden emplearse diferentes energías ya sea cobalto o electrones. La invasión estromal cervical profunda, la enfermedad metastásica en los ganglios regionales, la extensión a parametrios y los márgenes quirúrgicos positivos sirven como factores de riesgo para la reaparición de tumores cervicales La radioterapia postoperatoria adyuvante destinada a reducir la reaparición del cáncer de cuello uterino se administra a tumores de más de 4 $\mathrm{cm}$ con metástasis de ganglios linfáticos. ${ }^{(9-12)}$

La radioterapia debe administrarse de forma concurrente con la quimioterapia, utilizando fundamentalmente cisplatino por su efecto radiosensibilizante. Este es el método preferido de tratamiento, pues la quimioterapia inhibe el crecimiento de las células del cáncer de cuello uterino al prevenir su división activa y crecimiento. También pueden ser utilizados otros agentes como carboplatino, metotrexato, paclitaxel y topotecan. ${ }^{(13)}$ Aunque es capaz de tratar el cáncer de cuello uterino avanzado que se ha diseminado a otros órganos, esta forma de terapia está limitada por sus efectos secundarios perjudiciales. (14) Varios estudios han ilustrado una mayor tasa de supervivencia en mujeres tratadas con quimioterapia seguida de radiación, como resultado de la selección de células tumorales resistentes cruzadas que retrasan el inicio de la terapia curativa. La principal limitación de los medicamentos quimioterápicos es su incapacidad para distinguir las células cancerosas de sus contrapartes no cancerosas; por lo que estos también inhiben el funcionamiento de las células sanas. ${ }^{(15)}$

Los tumores neuroendocrinos son agresivos y se desarrollan a partir de células endocrinas en varios órganos, incluido el tracto genital femenino, y específicamente el cuello uterino. Representan entre el $0,9 \%$ y el $1,5 \%$ de todos los tumores del cuello uterino y generalmente afectan a las mujeres en edad reproductiva. Dada la rareza de la enfermedad, actualmente se dispone de datos limitados sobre la biología, el comportamiento clínico y el manejo de estos tumores agresivos. Como resultado, no hay pautas de tratamiento, basadas en ensayos clínicos prospectivos y bien diseñados actualmente disponibles, y los diferentes enfoques multimodales se derivan principalmente de tumores neuroendocrinos del pulmón. Por estas razones, los tumores 
neuroendocrinos representan un desafío terapéutico considerable para los oncólogos ginecológicos en todo el mundo. Los esfuerzos en la recolección y análisis sistemática de series relevantes de pacientes con tumores neuroendocrinos pueden constituir un verdadero paso hacia una mejor comprensión y tratamiento de estos tumores agresivos. Compartir estos esfuerzos y conocimientos puede ayudar a los ginecólogos oncólogos de todo el mundo a afrontar mejor esta "batalla desafiante". ${ }^{(16)}$

\section{Metodologia.}

Varias bases de datos de PubMed y la Biblioteca Nacional de Medicina fueron buscadas de 2015 a 2020 para estudios en todo el mundo que fueron publicados en inglés y traducidas al español. Después de examinar todos los resúmenes de las publicaciones identificadas por la búsqueda inicial, se incluyeron en el análisis estudios e informes de casos sobre mujeres con SCCC. La idoneidad de los estudios se definió a los efectos de esta revisión como la notificación de las características clínicas o biológicas, el tratamiento o los resultados clínicos de los pacientes con SCCC.

\section{Resultados.}

Paciente mestiza, femenina, de 58 años que en el 2014 se diagnosticó con carcinoma infiltrante no queratinizante de células pequeñas de cérvix, clasificada etapa IB2 y se trató con quimioterapia neoadyuvante esquema Cisplatino/Etopósido por 6 ciclos. Operada el 17/7/2014 cuya metodología fue histerectomía total con doble anisectomía y disección ganglionar inguinal bilateral; la biopsia informó: endometrio, miometrio, trompas, ovarios dentro de límites normales y 7 ganglios no metastásicos. Recibió tratamiento adyuvante con Radioterapia (modalidades teleterapia y braquiterapia) que terminó el 4-12-2014. Se mantuvo controlada en seguimiento durante 2 años y en junio del 2016 asistió a consulta con deposiciones diarreicas sanguinolentas en número de 4-5 al día con flemas, restos de tejido mucoso, tenesmo y pujos, acompañado de palidez cutáneo- mucosa, frialdad, sudoración, astenia y disuria. En región perianal se constató piel con cambios de coloración y textura indurada. Los complementarios mostraron resultados consistentes en $5 \mathrm{~g} / \mathrm{l}$ de hemoglobina y sangre oculta en heces fecales positiva. Se realizó otros estudios como:

US abdomen y ginecológico (9-5-16): normal.

Colonoscopia (30-6-16) proctitis actínica severa, colitis transversa actínica ligera, divertículo colon descendente y pólipo orificio anal interno.

Cistoscopia con biopsia (1-10-16): cistitis hemorrágica con cambios epiteliales y estromales inducidos por la radiación. 
Mantuvo igual sintomatología y se añadió dolor inguinal izquierdo mantenido de moderada a severa intensidad a pesar de tratamiento sintomático.

US partes blandas región glútea (24-1-17): cerca de pliegue Interglúteo izquierdo área de baja ecogenicidad de $62 \times 28 \mathrm{~mm}$ a $6 \mathrm{~mm}$ de piel.

TAC (20-2-17): engrosamiento de $13 \mathrm{~mm}$ de la pared anterior del recto, lesión osteoblástica de aspecto secundario en articulación sacroilíaca izquierda.

Gammagrafía ósea (3-4-17): captación de radiofármaco a nivel de L5, articulación sacroilíaca y coxofemoral izquierda.

CAAF glúteo izquierdo (7-3-17): negativo de células neoplásicas.

Dada la severidad y persistencia de las manifestaciones clínicas y los hallazgos encontrados se decidió realizar tratamiento con quimioterapia esquema ciclofosfamida/ adriamicina/ vincristina por 6 ciclos y ácido zoledrónico. Además de tratamiento de soporte con epocim, ácido tranexámico y azulfidina.

Evolucionó satisfactoriamente con recuperación clínica importante, se mantiene con ECOG 0, buena ganancia de peso, coloración de piel y mucosa normal, sin dolor. En enero del 2018 se constató lesión vegetante periuretral de la cual se tomó biopsia que informó epitelio escamoso con displasia leve y metaplasia escamosa queratinizante. Actualmente viva, y presenta calidad de vida.

\section{Discusión}

Las neoplasias neuroendocrinas (NEN) son neoplasias malignas agresivas derivadas de células neuroendocrinas. El término neuroendocrino se refiere al hecho de que las células tumorales se originan en el neuroectodermo embrionario y muestran un perfil inmunohistoquímico consistente con las células glandulares endocrinas. ${ }^{(17)}$ Pueden o no secretar hormonas peptídicas. En los seres humanos, las NEN se encuentran típicamente en el tracto gastrointestinal, el páncreas y los pulmones y se subdividen en NEN bien diferenciadas y NEN mal diferenciadas. ${ }^{(18)}$ Las NEN bien diferenciadas incluyen tumores neuroendocrinos (NET) G1 (también conocidos como carcinoides típicos), NET G2 (también conocido como carcinoides atípicos) y NET G3. Las NEN mal diferenciados incluyen NEN de células pequeñas y NEN de células grandes. En raras ocasiones, las NEN también pueden ocurrir en otros órganos como el tracto genital femenino tal y como se presentó en este caso. ${ }^{(19)}$

Debido a la rareza de esta neoplasia maligna, el manejo del SCCC es difícil y asociado con la incertidumbre. Es necesario un enfoque interdisciplinario, ya que la mayoría de los estudios que investigan el tratamiento de tumores neuroendocrinos se han realizado 
en pacientes con tumores en órganos distintos del cuello uterino, principalmente el pulmón y el páncreas. ${ }^{(20)}$

Aunque los carcinomas de células pequeñas pueden surgir en casi cualquier órgano, aproximadamente el 95\% surgen en el pulmón; por lo tanto, las raras excepciones, que incluyen el carcinoma de células pequeñas del cuello uterino, se conocen como carcinomas extrapulmonares de células pequeñas. ${ }^{(21)}$ Estas neoplasias muestran un perfil inmunohistoquímico consistente con la diferenciación neuroendocrina y pueden expresar cromogranina A, sinaptofisina, CD56 (N-CAM), PGP9.5 y NSE. Estos inmunomarcadores, en la actualidad son obligatorios para el diagnóstico. ${ }^{(22)}$

Los carcinomas de células pequeñas del cuello uterino se observan principalmente en mujeres más jóvenes en edad reproductiva. La asociación etiopatogénica entre el carcinoma de células pequeñas cervicales y las infecciones por VPH de alto riesgo ha sido bien documentada en algunos estudios. ${ }^{(23)}$ Un meta- análisis que incluye más de 30.000 cánceres cervicales invasivos reveló que el VPH 16 (59\%), 18 (13\%), 58 (5\%), $33(5 \%)$ y $45(4 \%)$ fueron los subtipos más frecuentes en los carcinomas de células escamosas cervicales. VPH 18 (37\%), 16 (36\%), 45 (5\%), 31 (2\%) y $33(2 \%)$ fueron los más frecuentes en adenocarcinomas cervicales. Muchos estudios han establecido que la prevalencia de diferentes tipos de VPH de alto riesgo en el carcinoma de células pequeñas cervicales osciló entre el 50 y el 100\%, y que el VPH 18 puede ser el tipo más frecuente. ${ }^{(24)}$

El carcinoma de células pequeñas del cuello uterino tiene un pronóstico mucho peor en comparación con otros tipos histológicos de carcinoma cervical, como el carcinoma de células escamosas del cuello uterino (CCS), el adenocarcinoma o el carcinoma de células adenoescamoso; se informó que la supervivencia en la etapa I- IIA, IIB- IVA y IVB a 5 años de enfermedad era del 36,8\%, 9,8\% y $0 \%$, respectivamente. ${ }^{(25)}$

No se disponía de datos prospectivos para comparar la cirugía con la radioterapia primaria para el SCCC resecable. Chen y otros informaron previamente una menor tasa de fracaso locorregional en pacientes que recibieron radioterapia primaria que aquellos que se sometieron a cirugía primaria en la etapa I-II SCCC (6\% vs. 27\%, P a 0.009). Cohen y otros encontraron que la supervivencia global mejoró en pacientes que recibieron histerectomía radical $(38,2 \%)$ frente a aquellos que recibieron radioterapia $(23,8 \%){ }^{(26,27)}$

Las modalidades de tratamiento del SCCC y del cáncer de pulmón de células pequeñas son similares debido a un comportamiento biológico similar, incluyendo la afectación de los ganglios linfáticos, la invasión vascular y la recurrencia temprana. La quimioterapia es un componente importante de la terapia multimodal. Algunos estudios identifican la quimioterapia como parte integral del tratamiento para todas las etapas de 
la enfermedad. Mientras que otros han encontrado que la quimioterapia simultánea o la quimioterapia adyuvante mejoraron la supervivencia en el SCCC. ${ }^{(28)}$

En la actualidad, todavía no hay consenso sobre qué modalidad debe ser el tratamiento de primera línea. ${ }^{29)}$ Con frecuencia se han utilizado regímenes de EP (etopósido y cisplatino) o CAV (ciclofosfamida, adriamicina y vincristina). Como resultado de los estudios, el SCCC siempre ha sido tratado con una combinación de cirugía, quimioterapia y/o RT. Sin embargo, no hay consenso sobre la eficacia de los protocolos de tratamiento. La Sociedad de Oncología Ginecológica (OSI) recomienda que para los tumores neuroendocrinos cervicales de menos de $4 \mathrm{~cm}$, se debe realizar histerectomía radical con linfadenectomía, con la opción de quimioterapia adyuvante. ${ }^{(30)}$

Los factores patológicos, como el margen de cirugía, la invasión linfovascular y la invasión parametrial, son los factores de pronóstico del cáncer de cuello uterino. El valor pronóstico de los factores patológicos anteriores en el SCCC sigue siendo controvertido. Un estudio de Wang y otros mostraron que los márgenes quirúrgicos positivos eran un factor pronóstico adverso para la supervivencia libre de recaída ( $\mathrm{P}<$ 0,001), pero no en la supervivencia específica del cáncer $(\mathrm{P}-0,593)$, la invasión linfovascular y la extensión parametrial no tuvieron ningún efecto de supervivencia en los análisis multivariados. Se ha informado que el tabaquismo y la etapa avanzada son factores de mal pronóstico para la supervivencia en pacientes con carcinoma neuroendocrino de células pequeñas del cuello uterino.

\section{Conclusiones.}

- Se trata de una entidad poco frecuente en esta localización $(0,9-1,5 \%)$ y son más frecuentes en mujeres jóvenes.

- Los elementos clínicos son similares a el carcinoma de cérvix de histología convencional por lo que siempre se requiere de estudio inmunohistoquímico.

- Como resultado de los estudios, el SCCC siempre ha sido tratado con una combinación de cirugía, quimioterapia y/o RT.

\section{Referencias bibliográficas.}

Small W, Bacon MA, Bajaj A, Chuang LT, Fisher BJ, Harkenrider MM, et al. Cervical cancer: A global health crisis [Internet]. Vol. 123, Cancer. John Wiley and Sons Inc.; 2017 [citado 11 de noviembre de 2020]. p. 2404-12. Disponible en: https://pubmed.ncbi.nlm.nih.gov/28464289/ 
Bray F, Ferlay J, and Soerjomataram I, et al (2018) Global cancer statistics 2018: GLOBOCAN estimates of incidence and mortality worldwide for 36 cancers in 185 countries CA Cancer J Clin 68 394-424 https://doi.org/10.3322/caac.21492 PMID: 302075933.

Koh WJ, Abu-Rustum NR, and Bean S, et al (2019) Cervical cancer, version 3.2019, NCCN clinical practice guidelines in oncology J Natl Compr Canc Netw 17 6484 https://doi.org/10.6004/jnccn.2019.0001 PMID: 306591314.

Akinyemiju T, Ogunsina K, and Sakhuja S, et al (2016) Lifecourse socioeconomic status and breast and cervical cancer screening: analy- sis of the WHO Study on Global Ageing and Adult Health (SAGE) BMJ Open 11 1275-1281 10.

Momenimovahed Z, Tiznobaik A, and Taheri S, et al (2019) Ovarian cancer in the world: epidemiology and risk factors Int J Women's Health 11 287-294 https://doi.org/10.2147/IJWH.S197604 11.

Ginindza TG, Dlamini X, and Almonte M, et al (2017) Prevalence of and Associated Risk Factors for High Risk Human Papillomavirus among Sexually Active Women, Swaziland PLoS One 12 1371-1389 https://doi.org/10.1371/journal.pone.0170189.

Derks M, van Lonkhuijzen LR, and Bakker RM, et al (2017) Long-term morbidity and quality of life in cervical cancer survivors: a multi- center comparison between surgery and radiotherapy as primary treatment Int J Gynecol Cancer 27 350-356 https://doi.org.

Fader AN (2018) Surgery in cervical cancer N Engl J Med 379 1955-1957 https://doi.org/10.1056/NEJMe1814034 PMID: 30379600 PMCID: 698903067.

Hofsjo A, Bohm-Starke N, and Blomgren B, et al (2017) Radiotherapy-induced vaginal fibrosis in cervical cancer survivors Acta Oncol 56 661-666 https://doi.org/10.1080/0284186X.2016.1275778 PMID: 28084859.

Liontos M, Kyriazoglo A, and Dimitriadis I, et al (2019) Systemic therapy in cervical cancer: 30 years in review Crit Rev Oncol Hematol 137 9-17 https://doi.org/10.1016/j.critrevonc.2019.02.009 PMID: 31014518.

Vordermark D (2016) Radiotherapy of cervical cancer Oncol Res Treat 39 516-520 https://doi.org/10.1159/000448902 PMID: 27614991.

Murtono M, Ndii MZ, and Sugiyanto S (2019) Mathematical model of cervical cancer treatment using chemotherapy drug Biol Med Nat Prod Chem 8 11-15 https://doi.org/10.14421/biomedich.2019.81.11-15 72. 
Ilancheran A (2016). Neoadjuvant chemotherapy in cervical cancer in pregnancy Best Pract Res Clin Obstet Gynaecol 33 102-107 https:// doi.org/10.1016/j.bpobgyn.2015.10.008.

Kong SY, Huang K, and Zeng C, et al (2018) The association between short-term response and long-term survival for cervical cancer patients undergoing neoadjuvant chemotherapy: a system review and meta-analysis Sci Rep 8 1-8 https://doi.org/10.1038/s41598-.

Aletti G, Laffi A. Neuroendocrine tumors of the cervix: An urgent call for joining forces. Vol. 29, International Journal of Gynecological Cancer. BMJ Publishing Group; 2019. p. 985.

Gadducci A, Carinelli S, Aletti G. Neuroendrocrine tumors of the uterine cervix: a therapeutic challenge for gynecologic oncologists. Gynecol Oncol. 2017;144:637-46. https://doi.org/10.1016/j.ygyno.2016.12.003.

Kim JY, Hong SM, Ro JY. Recent updates on grading and classification of neuroendocrine tumors. Ann Diagn Pathol. 2017;29:11-6. 3.

Guadagno E, de RG, de Del Basso Caro M. Neuroendocrine tumours in rare sites: differences in nomenclature and diagnostics-a rare and ubiquitous histotype. $\mathbf{J}$ Clin Pathol. 2016;69:563-74. https://doi.org/10.1136/jclinpath- 2015-203551.

Grande E, Capdevila J, Castellano D, Teulé A, Durán I, Fuster J, et al. Pazopanib in pretreated advanced neuroendocrine tumors: a phase II, open-label trial of the Spanish task force Group for Neuroendocrine Tumors (GETNE). Ann Oncol. 2015;26:1987-93. htt.

Zheng X, Liu D, Fallon JT, et al. Distinct genetic alterations in small cell carcinoma from different anatomic sites. Exp Hematol Oncol. 2015;4:2.

Bellizzi, A.M. Immunohistochemistry in the diagnosis and classification of neuroendocrine neoplasms: What can Brown do for you? Hum. Pathol. 2020, 96, 8-33. [CrossRef] [PubMed].

Small W Jr, Bacon MA, Bajaj A, Chuang LT, Fisher BJ, Harkenrider MM, Jhingran A, Kitchener HC, Mileshkin LR, Viswanathan AN, Gaffney DK. Cervical cancer: a global health crisis. Cancer. 2017;123: 2404-12.

Li P, Ma J, Zhang X, Guo Y, Liu Y, Li X, et al. Cervical small cell carcinoma frequently presented in multiple high risk HPV infection and often associated with other type of epithelial tumors. Diagn Pathol [Internet]. 22 de mayo de 
2018 [citado 11 de noviembre de 2020];13(1). Disponible en: https://pubmed.ncbi.nlm.nih.gov/29788963/

Egawa-Takata T, Yoshino K, Hiramatsu K, Nakagawa S, Serada S, Nakajima A, et al. Small Cell Carcinomas of the Uterine Cervix and Lung: Proteomics Reveals Similar Protein Expression Profiles. Int J Gynecol Cancer [Internet]. 1 de noviembre de 2018 [citado 11 de noviembre de 2020];28(9):1751-7. Disponible en: https://pubmed.ncbi.nlm.nih.gov/30358701/

Gibbs J, Mei S, Economos K, Lee YC, Kanis MJ. Clinicopathologic features, incidence, and survival trends of gynecologic neuroendocrine tumors: a SEER database analysis. Am J Obstet Gynecol. 1 de julio de 2019;221(1):53.e1-53.e6.

Wharton D, Kim E, Pagan J, Small W, Jaboin J, Ayala-Peacock D. Patterns of Care and Outcomes for Small Cell Carcinoma of the Cervix: A National Retrospective Analysis of 542 Cases. Adv Radiat Oncol [Internet]. 1 de mayo de 2020 [citado 11 de noviembre de 2020];5(3):412-8. Disponible en: http://www.ncbi.nlm.nih.gov/pubmed/32529135

Chen TC, Huang HJ, Wang TY, Yang LY, Chen CH, Cheng YM, Liou WH, Hsu ST, Wen KC, Ou YC, Hung YC, Lai HC, Ho CM, Chang TC. Primary surgery versus primary radiation therapy for FIGO stages I-II small cell carcinoma of the uterine cervix: A retrospective Tai.

Zhou J, Yang HY, Wu SG, He ZY, Lin HX, Sun JY, Li Q, Guo ZW: The local treatment modalities in FIGO stage I-II small-cell carcinoma of the cervix are determined by disease stage and lymph node status. Cancer Med 2016;5:11081115.

Pei X, Xiang L, Ye S, He T, Cheng Y, Yang W, Wu X, Yang H: Cycles of cisplatin and etoposide affect treatment outcomes in patients with FIGO stage I-II small cell neuroendocrine carcinoma of the cervix. Gynecol Oncol 2017;147:589-596.

Xu F, Ma J, Yi H, Hu H, Fan L, Wu P, et al. Clinicopathological Aspects of Small Cell Neuroendocrine Carcinoma of the Uterine Cervix: A Multicenter Retrospective Study and Meta-Analysis. Cell Physiol Biochem [Internet]. 1 de octubre de 2018 [citado 11 de noviembre de 2020];50(3):1123-39. Disponible en: https://pubmed.ncbi.nlm.nih.gov/30355937/

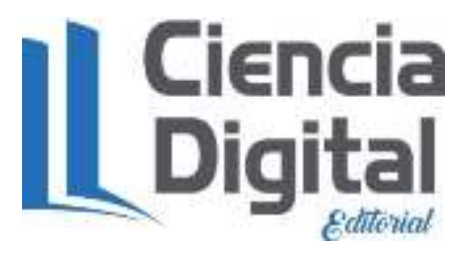


El artículo que se publica es de exclusiva responsabilidad de los autores y no necesariamente reflejan el pensamiento de la Revista Anatomía Digital.

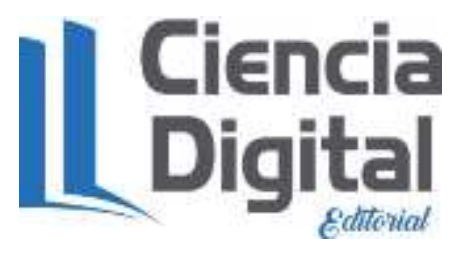

El artículo queda en propiedad de la revista y, por tanto, su publicación parcial y/o total en otro medio tiene que ser autorizado por el director de la Revista Anatomía Digital.
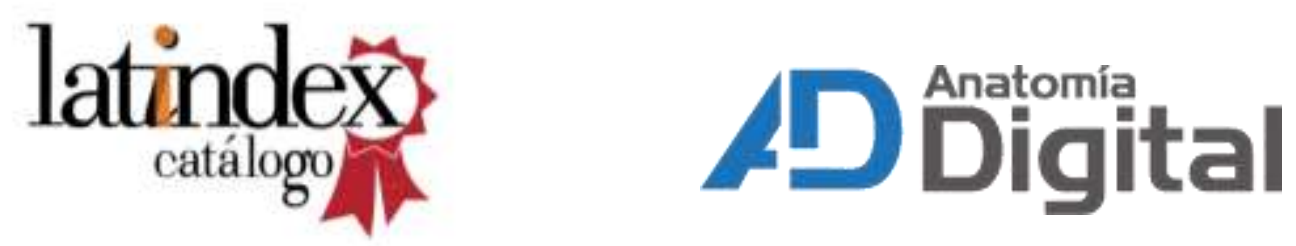

Indexacione:

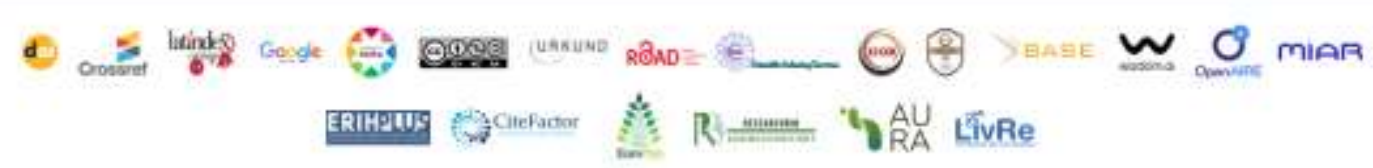

